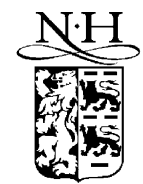

ELSEVIER
Mathematics and Computers in Simulation 58 (2002) 435-442

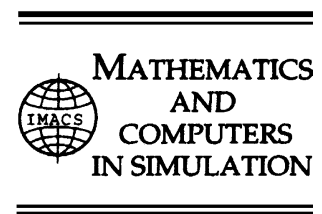

www.elsevier.com/locate/matcom

\title{
Noise-induced absolute instability
}

\author{
Sergey P. Kuznetsov \\ Institute of Radio-Engineering and Electronics, Russian Academy of Sciences, \\ Saratov Division, Zelenaya 38, Saratov 410019, Russia
}

\begin{abstract}
Noise-induced absolute instability is demonstrated in computer experiments with a one-dimensional reactiondiffusion system. This phenomenon may occur in spatially extended flow systems of different nature, when the parameters are chosen close to the point of transition from convective to absolute instability. (C) 2002 IMACS. Published by Elsevier Science B.V. All rights reserved.
\end{abstract}

Keywords: Noise-induced transition; Absolute instability; Convective instability; Pattern formation; Open flow

Studies of the effect of noise on bifurcations in non-linear dynamical systems have a long history [1]. For low-dimensional systems, this is now a well-established field, embracing such phenomena as noise-induced transitions, bifurcation precursors, and chaotic band merging (see, e.g. [2] for references and discussion). In particular, Crutchfield et al. [3] have shown how a gap of unobservable states develops in the period-doubling transition to chaos, and how the presence of noise obliterates the detailed structure in the invariant density. Scaling theories for the transition to chaos in the presence of noise have been worked out, e.g. by Crutchfield et al. [4] and by Shraiman et al. [5].

Less is known about noise-induced phenomena for non-equilibrium transitions in spatially extended systems (see, e.g. Cross and Hohenberg [6], Hohenberg and Swift [7], and Ahlers et al. [8] for a review and further references).

Formation of noise-stimulated patterns in a convectively unstable distributed system was studied, in particular, by Deissler [9,10] and Deissler and Farmer [11]. They considered the Ginzburg-Landau equation with flow and argued that the reason for the appearance of intermittent turbulent states, or coherent non-linear patterns, is that the system acts as a deterministic frequency-selective amplifier of noise from microscopic magnitude up to a level of relevant non-linearity. An analogous interpretation of pattern formation in reaction-diffusion systems with noise was suggested by Borckmans et al. [12]. In view of extreme sensitivity of the convectively unstable state to perturbations, the authors conclude that wave-patterns may be generated and sustained due to amplification of fluctuations from an upstream noise source. Similar problems were also studied in significant detail in connection with the so-called acousto-electric effect [13-15]. On the other hand, in recent works of Landa [16,17], the onset of turbulence

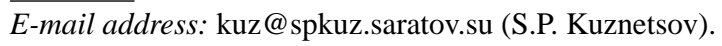


in flow systems is treated as a kind of noise-induced transition. This appears to suggest a more subtle interplay between fluctuations and dynamics than a straightforward amplification of noise.

Let us recall that an instability in a spatially extended system is termed absolute if a localized initial perturbation gives rise to growth of the amplitude observed at any fixed point in space. The instability is called convective if the growing perturbation drifts in such a way that observation at a fixed point in space exhibits a final decay of the amplitude to zero. The aim of this article is to show that near the threshold of a change of the instability from convective to absolute a specific kind of noise-induced transition in the open flow may occur.

In a previous publication [18], we presented an analysis of convective and absolute instabilities in a one-dimensional Brusselator flow model. The transition from one type of instability to another occurs at some critical flow rate, which depends on other parameters of the system. The transition is associated with a change in the direction of propagation of a front, which separates an undisturbed region upstream from a region with large amplitude of oscillations downstream. For flow rates below the critical value $c_{0}$, the front moves against the flow, and in the final state the whole volume of the system will be occupied by the oscillating patterns. For flow rates above $c_{0}$, the front is advected downstream and finally leaves the reactor space; then the system remains in a non-excited steady-state. The transition, which occurs at $c=c_{0}$, combines properties intrinsic to soft and hard bifurcations: it is abrupt (i.e. the amplitude jumps from a large level to zero, or vice versa, when the flow rate $c$ is slowly changed), but reversible $[18,19]$. Such a transition may obviously exhibit unusual peculiarities with respect to the influence of random fluctuations.

Let us discuss the effect of noise on this transition in the Brusselator flow model. (It may be hoped that the analysis is of common significance for different systems with convective or absolute instability in dependence on the direction of front propagation. Indeed, all relevant properties of the transition are linked just with the noise-induced motion of the front.)

In dimensionless variables the one-dimensional Brusselator flow model is governed by the following equations $[18,20]$.

$$
U_{t}+c U_{x}=A-B U-U+U^{2} V+\sigma U_{x x}, \quad V_{t}+c V_{x}=B U-U^{2} V+V_{x x}
$$

The chemical reactants are supposed to be supplied to one end of a lengthy reactor and flow down the reactor from $x=0$ to $L$ at a speed $c$. The dynamic variables $U(x, t)$ and $V(x, t)$ describe concentrations for two interacting chemical species, and $\sigma$ is the ratio of the diffusion constants for these reagents. $A$ and $B$ stand for two other species presented in excess and are considered as parameters.

The kinetic Eq. (1) provide for a single spatially uniform stationary solution of the form $U=A$ and $V=B / A$. Depending on $A$ and $B$ this solution may exhibit a Hopf bifurcation (leading to temporal oscillations) or a Turing bifurcation (producing spatial structures), and both of these instabilities may be either convective or absolute [18]. Fig. 1 shows the spatio-temporal dynamics of the model near the threshold of the absolute instability. Here, the system is started with an initial perturbation in the middle of the reactor, and noise-free equilibrium boundary conditions $\left.U\right|_{x=0}=A$ and $\left.V\right|_{x=0}=B / A$ are imposed at the upstream (left) end. A standard finite-difference method of the second order was used to obtain the numerical solution to the partial differential Eq. (1).

At the parameters $A=1.5, B=3.414392$, and $\sigma=0.25$, which correspond to Fig. 1, the critical velocity is $c_{0}=0.9$. For $c<c_{0}$ the Turing instability is absolute, and the front moves to the left. Its final stabilization at the upstream end of the reactor is clearly caused by the boundary conditions. For $c>c_{0}$ the instability is convective, the front moves to the right, and after a while it will leave the reactor 


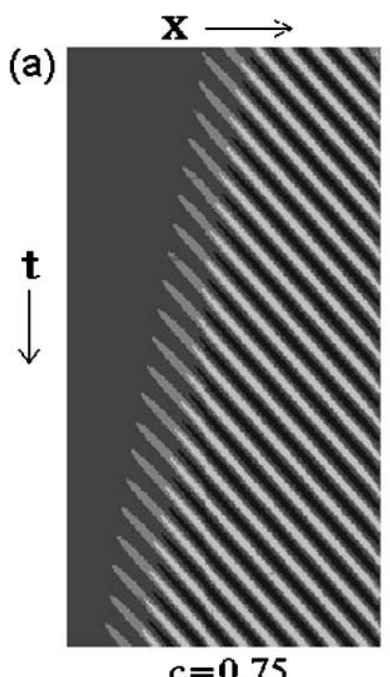

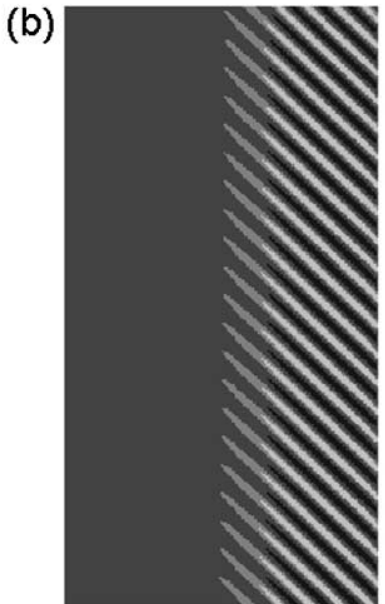

$c=0.9$

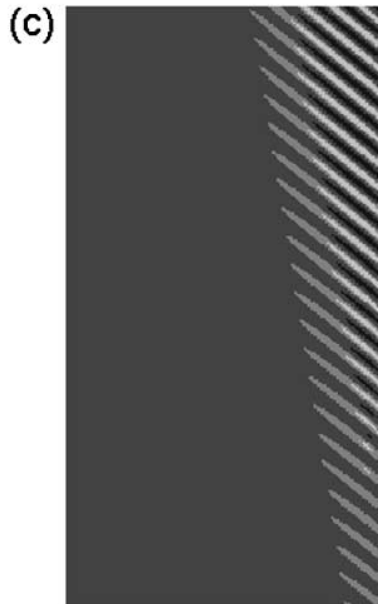

$c=1.05$

Fig. 1. Space-time diagrams for the Brusselator model of different flow rates: (a) absolute instability of Turing type for $c<c_{0}$; (b) threshold of the absolute instability $c=c_{0}$ and (c) convective instability $\left(c>c_{0}\right)$. Grey tones indicate levels of the variable $U(A=1.5 ; B=3.414392 ; \sigma=0.25$ and $L=40)$.

space. At the critical flow rate, $c=c_{0}$, there is a kind of neutral stability: A front can be maintained near the middle of the reactor and stay there for a very long time. In the active region to the right of the front, observation at any specific point reveals in this case temporal oscillations of a period $T \cong 4.2$ as subsequent Turing stripes pass by with the flow.

To estimate carefully the current location of the front the following procedure is suitable. While performing the numerical integration of Eq. (1) over one full time-period $T$, the maximum value of $U(x, t)$ is estimated at each point in space to obtain the envelope function

$$
W(x)=\max (U(x, t)), \quad t \in(k T,(k+1) T) .
$$

The position in space, where this envelope function assumes some definite intermediate value $w_{0}$, is regarded as the front position. Now one can study the motion of the front in discrete time $k$.

Here we come to the main point of the study. Let us introduce a noise source at the upstream end of the reactor. For the present investigation, the concrete nature of this noise will be not essential. In the discussed numerical experiments a random, non-correlated perturbation to the input value of $U$ was added at each time step of integration. The amplitude of the perturbation was taken to be uniformly distributed from $-\varepsilon / 2$ to $\varepsilon / 2$.

Fig. 2 shows the spatio-temporal behavior of the envelope function for different values of the flow rate in the presence of an upstream noise source. The different grey tones correspond to different intervals for $w(x)$, with fully developed wave patterns in the white zones to the right.

As one might expect, for a finite input noise the numerical simulations demonstrate that oscillatory wave patterns appear in the downstream part of the system, both for $c$ slightly below and slightly above the threshold. Moreover, although fluctuating, the position of the front stabilizes inside the reactor even for $c>c_{0}$. 

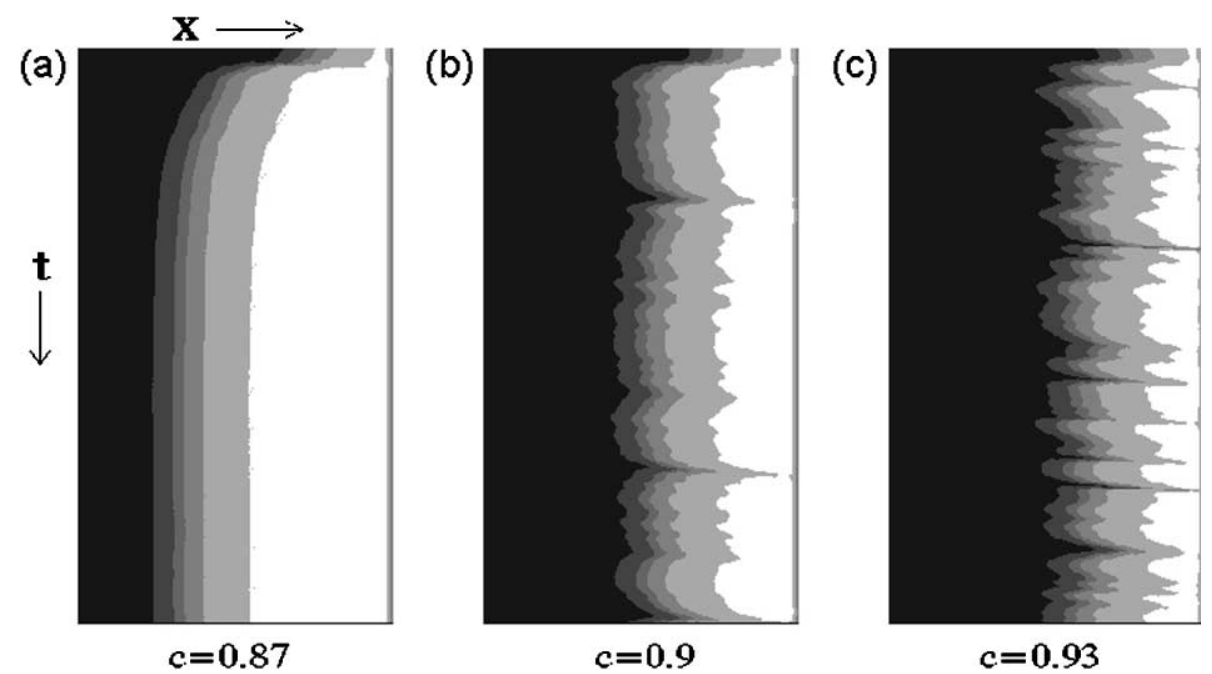

Fig. 2. Space-time diagrams for the Brusselator flow model in the presence of input noise. Grey tones indicate levels of the envelope function for $U$. Observe that the effect of noise can stabilize a front in the case of a convective instability $(c=0.93)$. Interval of the dimensionless time shown on each diagram is from 0 to 1764 . Input noise is of a fixed level, $\varepsilon=0.0005$. It is added at each time step $(\Delta t=0.042)$ as a random shift of $U$, at the input of the flow system: $U(0, n \Delta t)=A+\varepsilon \xi / 2$, where $\xi$ is a random variable distributed uniformly from -0.5 to 0.5 .

At this point of the analysis, the nature of the large amplitude oscillations in the white zone observed for $c>c_{0}$, is still not obvious (Fig. 2). Should we regard them simply as amplified noise, or are they the result of some noise-induced transition in the system? To clarify this point let us turn to additional numerical experiments.

Suppose first that the flow rate corresponds precisely to the threshold of absolute instability, and that the front is located somewhere inside the reaction space (sufficiently far from both ends). Having integrated Eq. (1) over one full time period of the oscillations we may estimate the new location of the front and find its displacement $\Delta x_{k}$. Then we shift the complete pattern $(U(x,(k+1) T), V(x,(k+1) T))$ to restore the initial position of the front and hereafter repeat the same procedure, time period after time period. In this way we may define a virtual front position $X=\sum \Delta x_{k}$ as the accumulated front shift. Obviously, with this procedure the fluctuations that impinge on the front act like a stationary random process.

Now the question is: How will the front coordinate $X$ evolve in time at the critical flow rate $c=c_{0}$ ? Intuitively, one might expect that by virtue of the neutral character of the stability, the motion of the front would be a random walk without any preferred direction. However, this is not the case. In the numerical experiments it is clearly observed that a directed motion of the front takes place, and the direction of motion corresponds to accumulated shifts towards the noise source (i.e. against the flow). Hence, to restore the condition of neutral stability in the presence of noise one must increase the flow rate. This is illustrated in Fig. 3: the virtual coordinate of the front is plotted as a function of time for different noise amplitudes. A closer analysis demonstrates that the front velocity is proportional to the square root of the noise amplitude $\varepsilon$. This amplitude dependence underlines the non-linear and statistical nature of the phenomenon. 

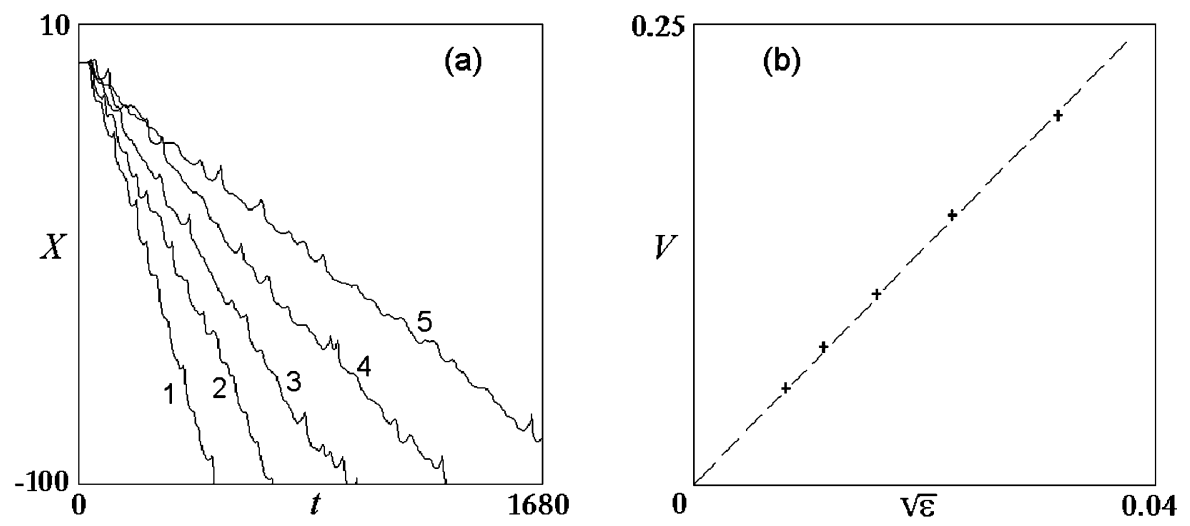

Fig. 3. Noise-induced drift of the fictive front coordinate $X$ (see text) at the threshold of absolute instability $(c=0.9)$. Panel (a) presents the front position as a function of time for different levels of the input noise. Curves $1-5$ correspond to $\varepsilon=0.001$, $0.0005,0.00025,0.000125,0.0000625$, respectively. Panel (b) gives evidence of a linear dependence of the induced front velocity $V$ on the square root of noise amplitude $\varepsilon$.

As previously noted, the strong sensitivity of the system to small amplitude noise arises because the noise signals are amplified as they are advected along with the flow. As a consequence, the effect of noise on the front dynamics is expected to increase with the distance between the noise source and the front. Such a dependence is indeed observed (see Fig. 4).

Fig. 5 shows the frequency characteristics for the amplification of a small amplitude Fourier component superimposed onto the convectively unstable, homogeneous state. Here, $c=0.93$, and the distance from

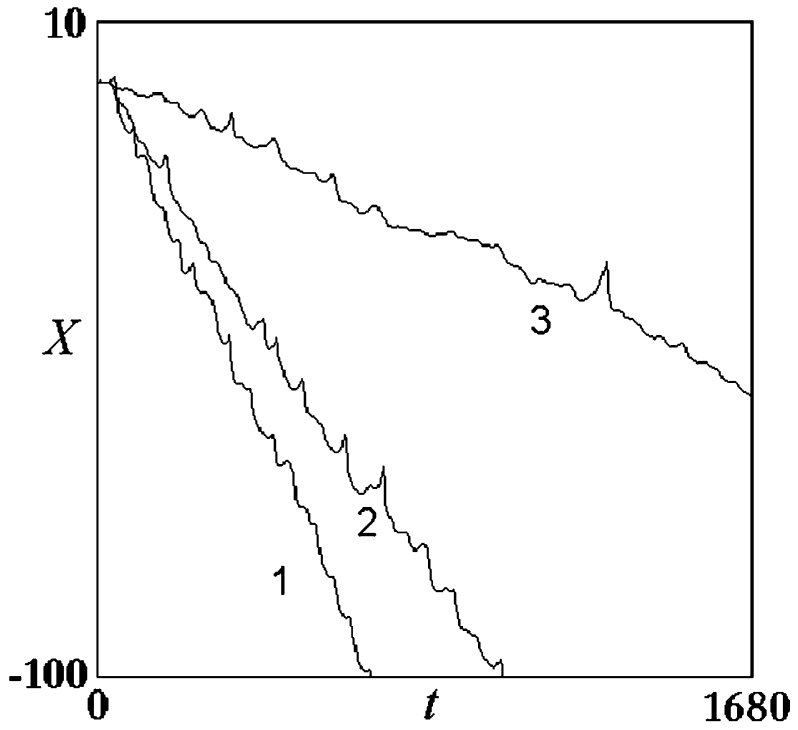

Fig. 4. Noise-induced drift of the fictive front coordinate $X$ at the threshold of absolute instability $(c=0.9)$. Curves $1-3$ correspond to different distances of the noise source from the front, 28.7, 23.7, and 18.7, respectively. The parameter of the noise intensity is fixed $(\varepsilon=0.0005)$. 


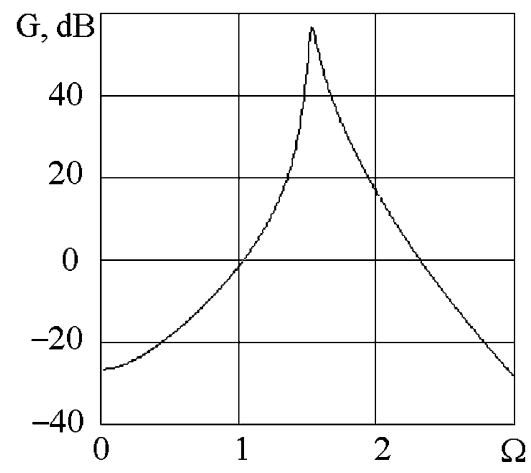

Fig. 5. Gain $G=20 \log \left(U_{\text {out }} / U_{\text {in }}\right)$ vs. frequency for Eq. (1) linearized near the convectively unstable equilibrium state, $A=1.5$, $B=3.414392, \sigma=0.25$, and $c=0.93$. Note the sharp peak at the frequency where the absolute instability will appear for slightly lower values of $c$.

the source to the observation point is $L_{0}=10$. Near the threshold of absolute instability, the amplification in the system is highly selective. The gain curve has a sharp peak at a frequency $f$ where the instability is going to appear. Let us assume that the front is far from the input end of the system. Then the noise, which effects the front dynamics, will have a very narrow frequency spectrum. In other words, the noise is characterized by a slowly decaying oscillatory tail of its correlation function.

To demonstrate significance of the long time correlations, another series of computer experiments was performed. A localized source of correlated noise was placed close to the front, and the induced virtual front velocity was determined in dependence of the correlation time (with a fixed mean square value of the amplitude of the noise source). For uncorrelated noise only a small random walk of the front coordinate $X$ takes place. On the other hand, for correlated noise a directed motion is observed, and the mean velocity $\langle V\rangle$ grows with the correlation time (Fig. 6).

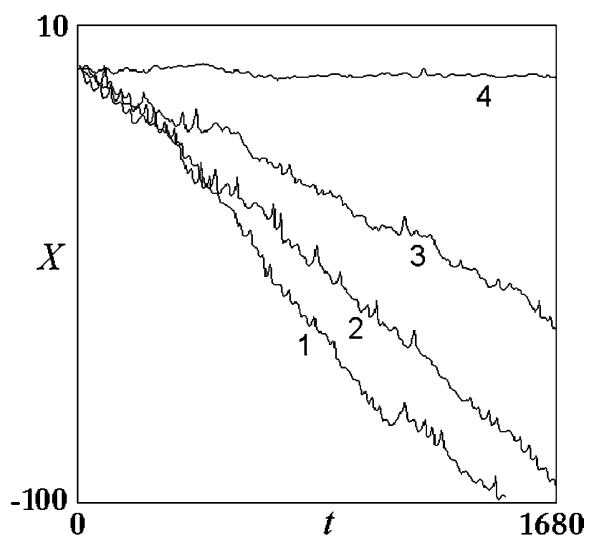

Fig. 6. Noise-induced drift of the fictive front coordinate $X$ in the case of a correlated noise source located close to the front. The spectrum of the noise source is Lorentzian with central frequency $2 \pi / T, T=4.2$. The spectral width is determined by the correlation time $t_{\mathrm{c}}$, and curves $1-4$ correspond to $t_{\mathrm{c}} / T=10,5,0.5$, and 0.01 , respectively. 
Based on these observations, we propose the following qualitative picture of the phenomenon that may be referred to as the noise-induced absolute instability.

First, let us consider instead of noise an input perturbation in a form of a wave-packet of mean frequency $f_{0}$. When the leading end of the wave-packet reaches the front, it may shift the front forth or back, depending on the phase relation between the incoming signal and the internally generated oscillations. However, gradually, while the packet arrives, the two phases will be tuned to one another (due to non-linear synchronization effects and due to the fact that perturbations arriving with the flow serve as priming for the front). Hence, the wave-packet will gradually interact with the front in a favorable phase, and if the duration of the packet is long enough, the final result will be a shift in the front towards the source.

In the case of a noise input, the fluctuations become narrow-band (or long-time correlated) as they are advected with the flow. Hence, the influence on the front may be regarded as the action of a random superposition of wave-packets, and the average effect will be a directed drift of the front towards the noise source. As the front approaches the source, the intensity of the impinging noise as well as its correlation time decrease. Hence, the velocity of the directed drift also decreases, and finally, the front stops at some finite distance from the noise source. Hereafter, it undergoes only random fluctuations near the mean position, as observed in Fig. 2.

If the velocity of the flow is slightly above the critical value $c_{0}$, the instability in the noiseless system is convective. In other words, the front propagates to the right and finally leaves the reaction space. In accordance with the above findings, the situation with a front moving against the flow may be restored if we introduce an input noise of sufficient magnitude. Then the spatio-temporal pattern characteristic for the case of absolute instability reappears, although with inevitable fluctuations.

The same phenomenon of noise-induced absolute instability may occur in other types of spatially extended flow systems as well. In particular, this concept may be useful for an initial understanding of the onset of turbulence in hydrodynamic systems with open flows $[16,17]$.

\section{Acknowledgements}

The work is performed under a partial support of CRDF, grant REC-006. Also the author acknowledges support for guest professorship from the Technical University of Denmark and thanks Prof. E. Mosekilde for discussions and help.

\section{References}

[1] L.S. Pontrjagin, A.A. Andronov, A.A. Witt, On the statistical treatment of dynamical systems, ZhETF 3 (1933) 165-180 (English translation: F. Moss, P.V.E. McClintock (Eds.), Noise in Non-linear Dynamical Systems, Vol. 1, Cambridge University Press, Cambridge, 1989, pp. 329-348).

[2] W. Horsthemke, R. Lefever, Noise Induced Phase Transitions, Springer, Berlin, 1984.

[3] J.P. Crutchfield, J.D. Farmer, B.A. Huberman, Fluctuations and simple chaotic dynamics, Phys. Reps. 92 (1982) $45-82$.

[4] J. Crutchfield, M. Nauenberg, J. Rudnick, Scaling for external noise at the onset of chaos, Phys. Rev. Lett. 46 (1981) 933-935.

[5] B. Shraiman, C.E. Wayne, P.C. Martin, Scaling theory for noisy period-doubling transitions to chaos, Phys. Rev. Lett. 46 (1981) 935-939.

[6] M.C. Cross, P.C. Hohenberg, Pattern formation outside of equilibrium, Rev. Mod. Phys. 65 (1993) 851-1112.

[7] P.C. Hohenberg, J.B. Swift, Effects of additive noise at the onset of Rayleigh-Bnard convection, Phys. Rev. A 46 (1992) 4773-4785. 
[8] G. Ahlers, C.W. Meyer, D.S. Cannell, Deterministic and stochastic effects near the convective onset, J. Stat. Phys. 54 (1989) $1121-1131$.

[9] R.J. Deissler, Noise-sustained structure, intermittency, and the Ginzburg-Landau equation, J. Stat. Phys. 40 (1985) 371-395.

[10] R.J. Deissler, External noise and the origin and dynamics of structure in convectively unstable systems, J. Stat. Phys. 54 (1989) 1459-1488.

[11] R.J. Deissler, J.D. Farmer, Deterministic noise amplifiers, Physica D 55 (1992) 155-165 .

[12] P. Borckmans, G. Dewel, A. De Witt, D. Walgraef, The differential flow instabilities, in: R. Kapral, K. Showalter (Eds.), Chemical Waves and Patterns, Kluwer Academic Publishers, Dordrecht, 1995.

[13] J.H. McFee, Transmission and amplification of acoustic waves in piezoelectric semiconductors, in: W.P. Mason (Ed.), Physical Acoustics, Academic Press, New York, 1966, pp. 1-45.

[14] N.I. Meyer, M. H. Jørgensen, Festkörperprobleme X (1970) 22-124.

[15] E. Mosekilde, Linear and Nonlinear Acoustoelectric Effects in Heavy Doped GaAs Epitaxial Single Crystals, The Technical University of Denmark, Lyngby, 1977.

[16] P.S. Landa, Noice-induced phase transitions and turbulence, in: Nonlinear Dynamics and Chaos, Applications to Physics, Biology and Medicine. ICND-96, Book of Abstracts, Saratov, 1996, p. 108.

[17] P.S. Landa, Turbulence in nonclosed fluid flows as a noise-induced phase transition, Europhys. Lett. 36(6) (6) (1996) 401-406.

[18] S.P. Kuznetsov, E. Mosekilde, G. Dewel, P. Borckmans, Absolute and convective instabilities in a one-dimensional Brusselator flow model, J. Chem. Phys. 106 (1997) 7609-7616.

[19] A. Couairon, J.M. Chomaz, Absolute and convective instabilities, front velocities and global modes in nonlinear systems, Physica D 108 (1997) 236-276.

[20] G. Nicolis, I. Prigogine, Self-Organization in Nonequilibrium Systems, Wiley, New York, 1977. 